\title{
Ancestors of the Lake. Art of Lake Sentani and Humboldt Bay, New Guinea deVirginia-Lee WEBB
}

\section{Gilles Bounoure}

\section{OpenEdition}

Journals

Édition électronique

URL : http://journals.openedition.org/jso/6456

DOI : 10.4000/jso.6456

ISSN : $1760-7256$

Éditeur

Société des océanistes

\section{Édition imprimée}

Date de publication : 15 décembre 2011

Pagination : 443-446

ISBN : 978-2-85430-031-4

ISSN : 0300-953x

\section{Référence électronique}

Gilles Bounoure, "Ancestors of the Lake. Art of Lake Sentani and Humboldt Bay, New Guinea deVirginiaLee wEBB 》, Journal de la Société des Océanistes [En ligne], 133 | 2e semestre 2011, mis en ligne le 31 décembre 2011, consulté le 24 septembre 2020. URL : http://journals.openedition.org/jso/6456 ; DOI : https://doi.org/10.4000/jso.6456

Ce document a été généré automatiquement le 24 septembre 2020.

(c) Tous droits réservés 


\title{
Ancestors of the Lake. Art of Lake Sentani and Humboldt Bay, New Guinea deVirginia-Lee WEBB
}

\author{
Gilles Bounoure
}

\section{RÉFÉRENCE}

WЕBB Virginia-Lee (ed.), 2011. Ancestors of the Lake. Art of Lake Sentani and Humboldt Bay, New Guinea, Houston, Menil Foundation. 136 pages, bibliogr., carte, index, 48 planches, 41 figures dans le texte.

1 Comme le soulignent dans leurs avant-propos Joseph Helfenstein et $\mathrm{Kr}$. Van Dyke, respectivement directeur et conservatrice de la collection de Menil, l'exposition dont cet ouvrage constitue le catalogue, présentée à Houston (Texas) du 6 mai au 28 août, puis au Sainsbury Centre for Visual Art de Norwich (Norfolk) du 27 septembre au 11 décembre 2011, aura offert au public américain (puis britannique) la première occasion de prendre contact avec les arts du lac Sentani depuis la manifestation que leur avaient consacrée Simon Kooijman et Douglas Newton au Museum of Primitive Art de New York en 1959-60, voilà plus d'un demi-siècle. Telle était aussi la provenance des deux premiers objets extra-européens que John et Dominique de Menil acquirent à Paris, en 1932, «heureuses prémisses» («serendipitous beginnings", écrit Kr. Van Dyke) précédant de plus d'une décennie leur décision de constituer la précieuse collection qu'expose et met en valeur désormais la fondation qu'ils ont créée. Après ces deux maro (panneaux de tissu d'écorce battue, bruts ou peints comme ici, $n^{\circ} 44$ et 46 du catalogue) que leur avait procurés Jacques Viot, ils parvinrent à acheter, en 1957 et 1960, trois sculptures monumentales ( ${ }^{\circ} 11,4$ et 21) provenant de sa collecte de 1929. De leur côté, dès 1939, Robert et Lisa Sainsbury avaient acquis une autre de ces sculptures $\left(n^{\circ} 24\right)$ qui demeure l'un des fleurons de leur collection conservée et exposée à Norwich. 
2 Dans l'introduction (pp. 12-19) où elle résume et met en perspective les contributions qu'elle a rassemblées, V.-L. Webb insiste sur la fascination exercée auprès des artistes surréalistes par les maro et les sculptures que Viot collecta en 1929 pour le compte du galeristeparisien Pierre Loeb ${ }^{1}$, et revient sur l'exposition précitée du Museum of Primitive Art, à laquelle J. de Menil avait prêté son concours. Dans «Lake Sentani Art. Connecting Ancestors and Descendants, Chiefs and Community» (pp. 22-29), D. Smidt expose les principaux aspects symboliques des motifs et décors sculptés sur les objets en bois de cette société lacustre, dont il décrit très sommairement l'organisation. L'essai de D. van Duuren, «Exploring the Lake Sentani art collections» (pp. 30-37), évoque les débuts de la pénétration occidentale et des collectes ethnographiques dans la région du lac avant de montrer comment s'en sont enrichies les collections publiques néerlandaises.

3 Les quatre dernières contributions portent sur des sujets plus circonscrits. Dans « It was like paradise. Paul Wirz at Lake Sentani and Humboldt Bay» (pp.38-47), A. E. Schmidt offre un portrait biographique sensible et nuancé du principal découvreur occidental de cette aire de style, notamment grâce à des extraits de correspondance familiale auxquels elle a eu accès. Avec "Jacques Viot and the Enchanted Castle " (pp. 48-55), Ph. Peltier s'attache à celui qui, après Wirz, réalisa la collecte la plus spectaculaire d'objets du lac Sentani, à son parcours personnel et à la réception de ces pièces une fois arrivées et exposées à Paris, dans le contexte artistique et intellectuel du début des années 1930 qui vit s'affirmer l'ethnologie en France. "Paintings Past, Present and Future» (pp.56-63) d'A.-K. Hermkens étudie l'évolution des maro et de leurs figurations depuis le plus ancien spécimen connu, collecté en 1858, jusqu'à nos jours où cet art jadis exclusivement féminin est devenu une activité principalement masculine. A.-K. Hermkens et M. Widjojo ont aussi recueilli, dans «Sentani Art and Artists Today. Reflections from Agus Ongge, Ferri Kaigere, and Martha Ohee » (pp. 64-67), le témoignage de trois créateurs contemporains, deux hommes et une femme, celle-ci marquant une énergie remarquable à restituer aux femmes un art du maro (ou plutôt du tapa peint) adapté aux demandes d'aujourd'hui.

4 À ces articles succède le catalogue proprement dit, qui reproduit et décrit vingt et une œuvres sur bois ainsi que quelques documents relatifs à leur collecte ou à leur réception, cinq photos dues à $\mathrm{P}$. Wirz et $\mathrm{J}$. Viot (après une vingtaine d'autres réparties dans le reste de l'ouvrage) et huit maro. Les notices établies par V.-L. Webb, qui recensent les circonstances de collecte, de conservation, de publication et d'exposition, ne sont qu'exceptionnellement accompagnées de commentaires à caractère principalement historique (à propos $d u$ «Lys », $\mathrm{n}^{\circ} 28$, et des vues qu'en a prises Man Ray, $n^{\circ} 29$ ) et ne proposent aucun élément d'interprétation ou d'identification, seraient-ce seulement les espèces animales représentées sur les maro et certaines œuvres sur bois ou le sexe des sculptures anthropomorphes (uniformément qualifiées de " figures »). Une seule de ces dernières a fait l'objet d'une datation au radiocarbone $\left(\mathrm{n}^{\circ} 13\right.$, «1790+/- 40 years »), les autres se trouvant attribuées pour la plupart aux XIX ${ }^{\mathrm{e}}$ $\mathrm{xx}^{\mathrm{e}}$ siècles, sans qu'on dispose apparemment d'informations sur les essences de bois mises en œuvre ni sur l'outillage de leurs créateurs, ce qui ne manque pas de surprendre pour des sculptures d'une telle rareté, et dont le prix, quand elles se trouvent sur le marché, exige généralement ce type d'examen ${ }^{2}$. Mais peut-être les responsables du catalogue et de l'exposition ont-ils voulu éviter à leur public ces détails intéressant surtout les spécialistes. 
5 Ces derniers ne peuvent manquer de comparer cet ouvrage aux publications existant déjà sur le sujet, et spécialement à celles de S. Kooijman $(1959,1992)$, largement plus détaillées, quoique en moins de pages, sur l'organisation sociale, l'évolution historique, le mode de vie, et les langues mêmes en usage sur les bords du Sentani (papoues) et aux alentours (austronésiennes), tous points sur lesquels ce catalogue est défaillant. Quoique " classiques », ni la question des affinités constatables entre les arts du Sentani et ceux du pays asmat, ni celle de leur possible source asiatique (Dongson) ou austronésienne (Lapita), discutées notamment par S. Kooijman (1992) et A.-K. Hermkens (1997, conclusions plus nuancées dans son texte de 2006) ne sont évoquées, non plus que les remarquables observations d'A.-M. et P. Pétrequin (2006, pp. 302 sq., 417-439) sur les industries et l'archéologie du bassin du Sentani, en lequel ils voient " une région clé en ce sens qu'elle représente aussi le point extrême d'arrivée vers l'est d'objets en bronze d'origine nusantarienne. »Ce qu'ils écrivent de la tradition potière féminine d'Abar, qui fournit tous les habitants du lac, confirme largement ce qu'a cherché à démontrer A.-K. Herm-kens (1997, 2009 et travaux intermédiaires) sur la stricte répartition sexuelle des tâches et des objets, sujet que le présent ouvrage n'aborde qu'à peine, à propos du maro comme ouvrage et vêtement féminin.

6 Sans doute est-ce au manque d'espace dans le catalogue et à diverses autres contraintes (lieux d'exposition, type de public attendu, etc.) qu'on devra imputer le caractère trop rapide de cette publication pour ce qui concerne «l'art du lac Sentani et de la baie de Humboldt » qu'elle prétend éclairer, sans réellement en faire son sujet principal. En volume, plus de la moitié des contributions est dévolue aux collecteurs et collectionneurs de ces arts, ou aux effets réels ou supposés de ce "collectionnisme» (en tant qu'accumulation ostentatoire d'objets d'art, d'après la définition courante) sur l'opinion publique ou les artistes occidentaux, à l'instar de Max Ernst qui se serait inspiré du « Lys » dans sa sculpture Les Asperges de la lune (1935), selon une fable récente (ici reprise p. 14), une autre le faisant plutôt s'inspirer là des statues ou des massues de l'île de Pâques! Du reste, les secrets perdurent. Si Ph. Peltier est parvenu à reproduire les instructions de P. Loeb à J. Viot pour ses collectes (document connu par ailleurs mais resté longtemps impubliable) et a pu aisément démystifier les allégations du voyageur sur les mœurs « sauvages » des riverains du Sentani ou la « disparition » de l'art du maro à Tobati sur la baie de Humboldt, il ne livre aucun détail nouveau sur cette collecte de 1929, parfois estimée au nombre peu croyable de soixante sculptures (Kooijman et Hoogerbrugge, 1992, p. 93), soit plus que celles que conservent les musées du monde entier.

7 Ainsi illustrée (et illustrant discrètement le parti pris de l'esthétique plutôt que de l'anthropologie), "l'histoire des collections » contribue certainement à auréoler ces objets d'un prestige que n'auraient pu leur conférer les données ethnographiques disponibles sur ces cultures sans cesse en évolution jusqu'à la fin du xix ${ }^{e}$ siècle, où elles importaient encore des sociétés voisines, comme celle de Vanimo plus à l'est, jusqu'à leurs initiations, leurs flûtes sacrées et leurs constructions cérémonielles. S. Kooijman observait lui-même (1959, p. 17) qu'en comparaison du nombre et de l'importance des importations observées chez les riverains du lac Sentani et de la baie de Humboldt, l'apport original de leur culture pouvait sembler petit, tout en restant incontestable, comme il tendait ensuite à le montrer. Était-ce ou non une difficulté à soumettre au public de cette exposition et aux lecteurs de ce catalogue, au risque de les détourner des œuvres présentées à leur admiration? On ne saurait attendre d'une publication de 
ce type, même due à des spécialistes aussi éminents, qu'elle réponde en tous points aux critères dits scientifiques, parfois opposés à ses exigences propres.

Quoique le bassin du Sentani ait été insuffisamment décrit du point de vue ethnographique, on peut attendre une meilleure connaissance de ses arts soit de recherches nouvelles, analogues à celles d'A.-M. et P. Pétrequin, soit d'informations anciennes jusqu'à présent négligées. Que penser par exemple de l'observation de la voyageuse, romancière, chroniqueuse et photographe Beatrice Grimshaw (1870-1953), qui, lors de son passage à Tobati en 1923, fut admise à pénétrer dans la maison des hommes ("Laki-laki house », écrit-elle en anglo-malais) où elle affirme avoir vu des masques de danse (1931, pp. 157-158, reformulation de son article de 1925) dont la trace ne semble pas s'être autrement conservée ? Quelques critiques qu'appellent aujourd'hui ses très abondants écrits, aucun des spécialistes qui les ont récemment examinés, $\mathrm{E}$. et H. Laracy (1977), S. Gardner (1985), Cl. McCotter (2007, parmi d'autres articles qu'elle lui a consacrés, non référencés ci-dessous) n'a relevé d'erreur flagrante dans ses reportages. Ici, nombre de ses informations, à l'instar du passé cannibale des gens de Tobati dont lui parla le chef du village, coïncident avec ce qu'avait appris Wirz de son côté. Si l'existence de ces masques, qu'elle n'a malheureusement pas photographiés, se trouvait corroborée, on pourrait y voir un autre indice d'importation culturelle dans la baie de Humboldt.

Quant aux sculptures elles-mêmes, fleurons de cette exposition et de son catalogue, les a-t-on suffisamment interrogées ? Leur destination reste obscure, et avec elle le sens à donner à leur figuration. On pourra s'étonner qu'un des principaux éléments de réponse dépende toujours de la communication orale que le directeur du Museum of Primitive Art, Robert Goldwater, aidant S. Kooijman à préparer son exposition, vint recueillir auprès de J. Viot au printemps 1959, trente ans après son passage sur les rives du Sentani (D. Smidt, pp. 25 et 29, n. 24 ; cf. Kooijman, 1959, p. 18). Selon son souvenir, ces statues anthropomorphes ornaient le sommet des pilotis, mais aussi les galeries et les passerelles des constructions établies sur le lac, ce que ne confirme aucune photographie d'époque. Pour sujet à caution que soit le témoignage de Viot, peu exact dans l'évocation de son séjour à Manokwari et à Kurudu, il faisait conclure à C. A. Schmitz (1971, pp.55-56), au terme d'une excellente description de leur «rigidité héraldique» due à une "conception frontale», qu'aucune des sculptures anthropomorphes du Sentani «ne devrait être considérée comme une représentation d'ancêtre, leur fonction étant purement ornementale. » Ainsi ne subsisterait-il aucune image des « ancêtres du lac » mis en avant dans cette exposition.

Restent quelques hypothèses que des examens plus attentifs permettraient de confirmer ou de balayer. Si ces sculptures, probablement chargées au moins de signaler la puissance des chefs, à l'instar des grandes jarres de sagou exposées à l'entrée de leurs demeures, appartenaient à l'architecture même de ces habitations lacustres, leurs créateurs avaient sans doute pris soin de les tailler dans le même «bois de fer » réputé imputrescible (Pometia sp., dont un des noms locaux est matoa) que les autres pilotis fichés dans le lac, plutôt que dans tel bois tendre comme le phuli mis en œuvre pour leurs canots. Il ne serait pas inutile non plus de chercher à déterminer si ces sculptures furent détachées des fûts qu'elles sommaient par les habitants du lac eux-mêmes, marquant ainsi un certain souci de conservation, ou si leurs dimensions actuelles résultent des coups de scie ou de hache réclamés par les collecteurs, Wirz, Viot et d'autres, pour les faire parvenir plus commodément en Occident. Voilà qui aiderait 
peut-être à comprendre comment ces figurations d'hommes et de femmes, décoratives ou «héraldiques» (selon le terme ambigu de C. A. Schmitz), s'intégraient dans les représentations de cette société si nettement divisée entre le masculin et le féminin, ou participaient éventuellement, comme cela a été constaté ailleurs, à des compétitions de prestige entre les chefs et les communautés qu'ils dominaient.

\section{BIBLIOGRAPHIE}

FRIEDE John, 2005. Catalogue of Objects, in Gregory Hodgins, Philippe Peltier, Dirk Smidt and Robert L. Welch, New Guinea Art. Masterpieces from the Jolika Collection of Marcia and John Friede, San Francisco, Fine Art Museums of San Francisco.

GARDNER Susan, 1985. For Love and Money. Beatrice Grimshaw's Passage to Papua, doctoral thesis, Rhode University.

GRIMSHAW Beatrice, 1925. The Sea Villagers of Humboldt's Bay, Wide World Magazine, 54, pp. 325-331.

-, 1931. Isles of Adventure. From Java to New Caledonia but Principally Papua, Boston-New York, Houghton Mifflin Company [1 ${ }^{\text {èr }}$ éd. 1930, Londres, Jenkins].

HERMKENS Anna-Karina, 1997. The Way of the Objects. Analogical inference and the Allocation of Meaning and Order in Lapita, Dongson and Lake Sentani Material Culture, MA thesis, Rijksuniversiteit Leiden (en ligne sur le site papuaweb).

-, 2006. La Région du lac Sentani, in Philippe Peltier et Floriane Morin (éds), Ombres de NouvelleGuinée. Arts de la grande île d'Océanie dans les collections Barbier-Mueller, Paris, Somogy, pp. 50-69.

,- 2009 . Gendered Objects. Embodiements of Colonial Collecting in Dutch New Guinea, The Journal of Pacific History 42, 1, pp. 1-20.

KoOIJMAn Simon, 1959. The Art of Lake Sentani, New York, The Museum of Primitive Art.

KOOIJMAN Simon et Jac HOOGERBRUGGE, 1992. Art of Wadke-Yamna Area, Humboldt Bay, and Lake Sentani, in Suzanne Greub (ed.), Art of Northwest New Guinea, from Geelvink Bay, Humboldt Bay, and Lake Sentani, New York, Rizzoli, pp. 57-125.

LARACY Eugenie and Hugh, 1977. Beatrice Grimshaw. Pride and Prejudice in Papua, The Journal of Pacific History 12, 3, pp. 154-175.

MCCOTTER clare, 2007. Woman Traveller/Colonial Tourist. Deconstructing the Great Divide in Beatrice Grimshaw's Travel Writing, Irish Studies Review 15-4, pp. 481-506.

PÉTREQUIN Anne-Marie et Pierre, 2006. Objets de pouvoir en Nouvelle-Guinée. Approche ethnoarchéologique d'un système de signes sociaux. Catalogue de la donation Anne-Marie et Pierre Pétrequin, Paris, Éditions de la Réunion des musées nationaux.

scHMITZ carl A., 1971. Oceanic Art. Myth, Man and Image in the South Seas, New York, Abrams. 